04

\title{
Метод определения скорости расширения канала на стадии электрического пробоя с помощью перехватывающего кольца с заземлением
}

\section{(C) В.В. Аполлонов, Н.В. Плетнев}

Институт общей фозики им. А.М. Прохорова РАН, 119991 Москва, Россия

e-mail: vapollo@kapella.gpi.ru; pletnev@kapella.gpi.ru

(Поступило в Редакцию 26 января 2016 г. В окончательной редакции 7 апреля 2016 г.)

\begin{abstract}
Описан метод, позволяющий исследовать динамику проводимости канала, образованного взрывом проволоки на стадии электрического пробоя. Приведены результаты экспериментов по определению скорости расширения канала проводимости протяженных (длиной до $\sim 1.9 \mathrm{~m}$ ) промежутков произвольной геометрической формы, образованных взрывающейся проволокой из меди диаметром $90 \mu \mathrm{m}$, при начальной запасенной энергии $2.7-3.7 \mathrm{~kJ}$ при напряжении $8-10 \mathrm{kV}$. Показано, что скорость расширения канала проводимости совпадает со скоростью распространения ударной волны и обратно пропорциональна радиусу ударной волны и времени ее распространения в степени $1 / 2$. Радиус ударной волны линейно зависит от времени ее распространения в степени $1 / 2$. Экспериментальные результаты удовлетворительно совпадают с расчетными значениями, приведенными S.C. Lin в модели сильной цилиндрической ударной волны. Показано, что диаметр канала проводимости определяется положением заднего фронта ударной волны.
\end{abstract}

\section{Введение}

При исследовании управляемого электрического пробоя $(\mathrm{EB})[1,2]$ в плазменном канале, образованном взрывающейся проволокой (EW) [3-8], способном пропускать ток в режиме короткого замыкания, необходимо знать температуру канала и его радиус [9]. Понятие радиуса канала относится к той его части, где в основном протекает ток во время главной стадии разряда [9]. Значение скорости расширения канала и его максимальный диаметр, соответствующий зоне с высокой проводимостью и температурой, важны при оценке удельного энерговклада и давления на фронте цилиндрической ударной волны, распространяющейся в атмосфере. В работах $[10,11]$ при исследовании канала искрового разряда в атмосфере для этой цели был применен теневой метод Теплера совместно с зеркальной разверткой методом вращающего зеркала. Канал искры определялся как токопроводящая область повышенной (практически полной) ионизации. Радиальное распространение этой области может быть обусловлено следующими процессами [10]:

1) теплопередачей, вызывающей расширение области высокой температуры, а следовательно, и высокой ионизации;

2) диффузией электронов и ионов в радиальном поле искры;

3) процессами фотоионизации.

Возникновение ударной волны со скоростью $\sim 1 \mathrm{~km} / \mathrm{s}$ требует скачка давления порядка десятка атмосфер [10]. Такой скачок не может быть вызван фотоионизацией даже в резко ограниченной области, так как давление за счет ионизации не может увеличиться в десятки раз [10].
Следовательно, в начальной стадии разряда должен происходить процесс, вызывающий резкий скачок давления, т. е. процесс взрывного типа [10,11]. В искровом разряде в течение времени $5 \cdot 10^{-7} \mathrm{~s}$ после пробоя на $1 \mathrm{~cm}$ длины канала выделяется энергия $\sim 0.1-1 \mathrm{~J}$, сравнимая с энергией, выделяемой при взрыве взрывчатых веществ. Таким образом, расширение канала действительно начинается взрывным процессом. В тонкой нити канала, образовавшегося при пробое, происходит резкое повышение температуры, а следовательно, и давления газа, что вызывает появление цилиндрической ударной волны, распространяющейся со скоростью, значительно больше скорости звука $a_{0}=340 \mathrm{~m} / \mathrm{s}$ (в невозмущенной атмосфере при комнатной температуре). Температура на фронте волны и непосредственно позади него, как показывают расчеты $\sim 10000 \mathrm{~K}$, обеспечивает интенсивную ионизацию канала [10]. По мере продвижения ударной волны температура на ее фронте понижается и, как показывают результаты оптического исследования по методу Теплера и его аналогов [10-12], фронт ударной волны отходит от границы канала. Дальнейшее развитие канала обусловлено движением газа как целого и постоянным выделением энергии тока в канале. Скорость расширения канала $[10,11]$ определяется величиной энергии $E(t)$, поступившей в канал к моменту $t$, и параметрами газа - плотностью $\rho$ и отношением удельных теплоемкостей $\gamma$ (при расчете которых существенно входят процессы ионизации и диссоциации газа).

В работе [12] исследовались световые явления, связанные с развитием канала искрового разряда. При удельной вложенной энергии $\sim 3 \mathrm{~J} / \mathrm{cm}$ границы синефиолетового (4700-3700 А) и инфракрасного свечений 
(7000-10000 $\AA$ ) распространяются с одинаковой скоростью только в начале разряда в течение первых $0.4-0.5 \mu$ s до достижения каналом диаметра $\sim 2.5 \mathrm{~mm}$. Момент резкого изменения в скорости перемещения границы коротковолнового излучения совпадает с первым максимумом тока в разрядном контуре. Сравнение развертки ударной волны и инфракрасного свечения [12] показывает, что граница этого свечения совпадает с границей заднего фронта ударной волны. Полученные снимки объясняют наличие резкого фронта у инфракрасного свечения за счет ограничения этого свечения уплотнением газа в ударной волне. Ударная волна характеризуется крутым передним фронтом, областью примерно одинаковой плотности и пологим задним фронтом, называемым иногда „оболочкой“ канала. Как показано в работе [12], процесс образования канала и его структуру можно представить следующим образом. За время нарастания тока в течение первой четверти периода колебаний происходит непрерывное увеличение энергии, выделяющейся в искре. При этом плазма разряда расширяется взрывообразно со сверхзвуковой скоростью. После достижения максимума тока поступление энергии в канал начинает уменьшаться, в результате чего происходит замедление в расширении плазмы и от его фронта отрывается ударная волна. Между плазмой, занимающей центральную часть разряда и излучающей сплошное свечение, и ударной волной образуется слой разогретого нейтрального газа, который дает ИК свечение в виде излучения дуговых линий.

В работе [13] рассматривались возможные причины наблюдаемого на опыте расширения канала искры во времени. По данным работы [14], электропроводность $\sigma$ воздушной плазмы резко возрастает с повышением температуры в пределах $T=(0.4-1.2) \cdot 10^{4} \mathrm{~K}$, достигая некоторого насыщения при температуре ионизации Тi $\geq 1.5 \cdot 10^{4} \mathrm{~K}[15]$. Независимо от конкретного механизма первичного нагрева воздуха до температуры Ті дальнейший его нагрев осуществляется джоулевой энергией разрядного тока, поскольку эффективная первичность механизма нагрева резко снижается. Скорость расширения канала рассчитывается на основе механизма первичного нагрева газа до температуры Ti, а величина джоулевой энергии канала может влиять на интенсивность этого процесса. Как утверждается в работе [13], ударный нагрев газа при максимальных скоростях движения фронта ударной волны $D_{b}=2-5 \mathrm{~km} / \mathrm{s}$ дает низкие температуры на фронте $T_{b}=(2-7) \cdot 10^{3} \mathrm{~K}<\mathrm{Ti}$. Поэтому граница канала искры, движущаяся с указанными скоростями, не может быть фронтом ударной волны. В модели, предложенной в работе [13], при температуре канала искры $T=(4-5) \mathrm{eV}$ максимум спектральной плотности энергии излучения приходится на длины волн $\lambda_{m}=(62-50) \mathrm{nm}$. Это излучение в большей части поглощается холодными слоями воздуха на границе канала, что ведет к их нагреву. Поглощение больших квантов света является также основным механизмом нагрева воздуха для последующей стадии развития искры, когда фронт ударной волны отделился от границы канала. При этом канал оказывается в области хвоста ударной волны, плотность газа в канале мала, а излучение становится оптически тонким. Ширина зоны лучистого прогрева в обоих случаях может быть оценена по коэффициенту поглощения $\chi_{v}=1 / l_{v}$ больших квантов света с энергией $h v$, равной энергии ионизации $J$ атомов воздуха [16]. Для условий в оболочке канала $T=1.2 \cdot 10^{4} \mathrm{~K}, N_{0}=5 \cdot 10^{18} \mathrm{~cm}^{-3}$ значение спектральной длины пробега нагревающих квантов света $l_{v}=1 / \chi_{v}=0.59 \cdot 10^{-2} \mathrm{~cm}$ [13]. Значение $l_{v}$ примерно на два порядка меньше радиуса канала искры, что позволяет рассматривать границу канала как тонкий слой поглощения света и ионизации. Как показывают расчеты в работе [13], электронная теплопроводность может играть существенную роль в области больших градиентов температуры на границе канала. Поток электронной теплопроводности поглощается на границе канала в слое толщиной $\Delta r \approx l_{v} \approx 0.6 \cdot 10^{-2} \mathrm{~cm}$, т.е. осуществляется одновременный совместный лучистый и теплопроводностный нагрев одного слоя газа на границе канала. В указанных случаях зона нагрева перемещается на новые слои холодного газа, что можно рассматривать как волновое движение некоторого тонкого фронта нагрева и ионизации - ионизационной волны. В работе [13] скорость фронта ионизационной волны в начальной стадии и в более поздние моменты времени расширения канала искры оценивалась из условия баланса энергии на фронте. Скорость расширения канала искры в начальной стадии составила $D_{\Sigma, \mathrm{e}}=1.14-2.77 \mathrm{~km} / \mathrm{s}$, причем роль электронной теплопроводности для рассматриваемого режима пренебрежимо мала. В более поздние моменты времени, например для $t=5 \mu \mathrm{s}$, скорость распространения ионизационной волны составляла $D_{\mathrm{v}, \mathrm{e}}=420 \mathrm{~m} / \mathrm{s}$. Здесь уже оказывается существенной электронная электропроводность, дающая основной вклад в значение скорости распространения фронта ионизационной волны.

В работе [17] приведены экспериментальные результаты, подтверждающие теорию S.C. Lin's [18] в модели сильной цилиндрической ударной волны. При взрыве цилиндрического заряда PETN (внешний радиус $15 \mathrm{~mm}$, внутренний радиус $4 \mathrm{~mm}$, высота $30 \mathrm{~mm}$ ) с помощью зонда ионизации и осциллографа была определена скорость ударной волны в зависимости от времени распространения или расстояния от поверхности заряда. Зависимость обратной величины скорости ударной волны $U^{-1}$ от времени $t^{1 / 2}$ для чисел Mach's в диапазоне 7-14 получилась линейной, что соответствует расчетным значениям в теории S.C. Lin's. Отклонение от линейной зависимости для чисел Мach's менее 3, предсказанное S.C. Lin, может быть обусловлено конечными размерами заряда взрывчатого вещества. Аналогичные экспериментальные результаты, подтверждающие теорию S.C. Lin's, в модели сильной цилиндрической ударной волны были приведены в работах F.D. Bennett's [19,20], 
где в качестве источника ударной волны был использован взрыв тонкой проволоки $\varnothing 90-160 \mu \mathrm{m}$, длиной $2 \mathrm{~cm}$ при запасенной энергии $118 \mathrm{~J}$.

В настоящей работе с помощью простой методики, не требующей оптических методов регистрации [10-12], определена зависимость скорости расширения канала проводимости, образованного взрывом медной проволоки, от времени при максимальной вложенной удельной энергии $\sim 1 \mathrm{~kJ} / \mathrm{m}$. В отличие от результатов, полученных в [17], в нашей работе при малых поперечных размерах взрываемой проволоки $\varnothing 90 \mu \mathrm{m}$ зависимость $U^{-1}$ от времени $t^{1 / 2}$ для чисел Mach's менее трех получилась линейной.

\section{Формирование управляемых электрических разрядов в канале, образованном взрывом проволоки и описание методики эксперимента}

В настоящей работе, также как и в экспериментах $[1,2]$ по созданию управляемого канала высокой проводимости в атмосфере, был использован вертикально ориентированный проводник из меди марки PEV-2, содержащей эмалевую оболочку, длина и диаметр, которого составляли: $L=133 \mathrm{~cm}, \varnothing 90 \mu \mathrm{m}$ при зарядном напряжении $U_{0}=8.6 \mathrm{kV}$ и емкости $C=74.8 \mu \mathrm{F}$. На рис. $1, a$ показана схема экспериментальной установки и положение датчиков для измерения тока поясом Роговского RL; напряжения на конденсаторной батарее c помощью делителя напряжения VD и положение торца полированного кварцевого волокна QF, передающее излучение из канала разряда на быстродействующий ріп фотодиод PD с временны́м разрешением $\sim 1 \mathrm{~ns}$. В отличие от схемы, представленной в работах $[1,2]$, на рис. 1, $a$ показан кольцевой электрод RE, подключенный к земле, который расположен симметрично по отношению к взрывающейся тонкой проволоке EFW вблизи анода на расстоянии $\sim 15 \mathrm{~cm}$ от него. В управляемом пробое в атмосферном канале, образованном взрывом проволоки (EW) при его расширении, часть разрядного тока замыкается на электрод RE. Зная внутренний диаметр кольца и интервал времени $\Delta t$ между началом пробоя и моментом замыкания (по осциллограмме тока) плазменного канала, можно определить среднюю скорость его расширения для данного радиуса кольца. Фотография перехватывающих колец из меди и дюраля с внутренними диаметрами от 19 до $36.5 \mathrm{~mm}$ приведена на рис. 1, $b$. Измерение формы импульса тока производилось с помощью дифференциального экранированного пояса Роговского с временны́м разрешением $\sim 30 \mathrm{~ns}$ и постоянной интегрирования $\theta=L_{\Sigma} / 2 R \approx 380 \mu \mathrm{s}$ [21]. Измерение формы напряжения на разрядном промежутке призводилось с помощью экранированного VD смешанного типа [22], подключенного к аноду батареи конденсаторов. Данный делитель напряжения дает ошибку измерения $\sim 1 \%$ при длительности фронта тока $\tau_{\phi} \geq 1 \mu \mathrm{s}[22]$. Более подробно

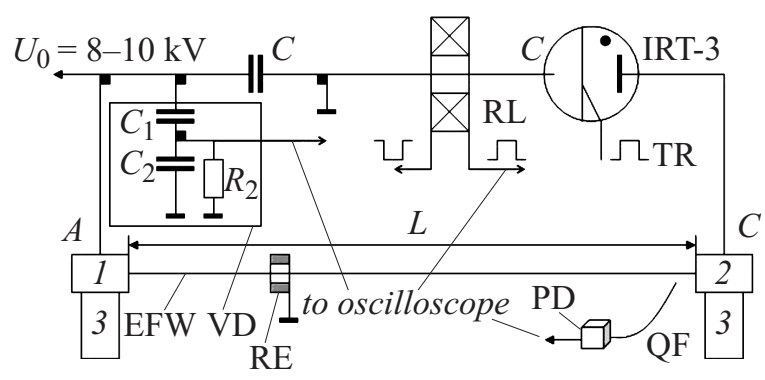

$a$

$b$

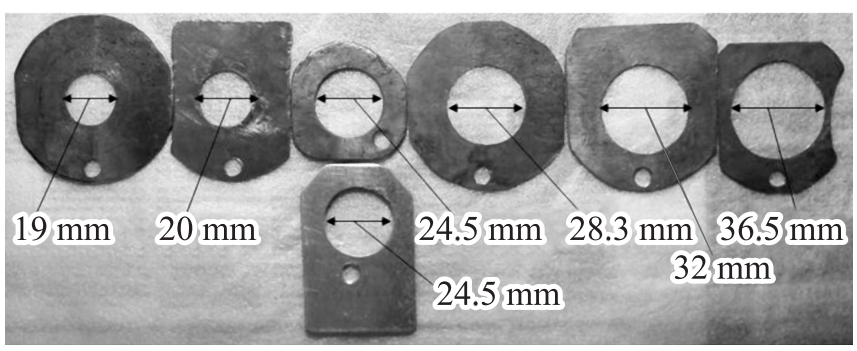

Рис. 1. Схема эксперимента по инициированию EВ в канале взорванной медной проволоки в вертикальном положении: 1,2 - латунные электроды, 3 - эбонитовые изоляторы, IRT-3 - игнитрон, коммутатор, RL - пояс Роговского, VD - делитель напряжения, TR - пусковой импульс, $\mathrm{EFW}$ - взрывающаяся тонкая проволока, PD - фотодиод, $\mathrm{QF}$ - кварцевое волокно, $A$ - анод, $C$ - катод, RE - перехватывающее кольцо $(a)$; фотографии перехватывающих колец из меди с диаметрами от 19 до $36.5 \mathrm{~mm}$ и одно кольцо из дюраля диаметром $24.5 \mathrm{~mm}(b)$.

работа делителя описана в работах [2,22]. Достоинством данного делителя является то, что он не шунтирует слаботочный источник зарядных напряжений. В качестве регистрирующего прибора в работе использовался цифровой осциллограф Tektronix TDS 220. Конденсатор емкостью $C=74.8 \mu \mathrm{F}$ заряжался от источника зарядных напряжений до значения $U_{0}=8.6 \mathrm{kV}$.

Общие принципы работы усовершенствованного нами источника зарядных напряжений описаны в работе [23]. Выходное напряжение источника регулируется плавно в пределах 2-12 kV. Максимальный зарядный ток $5 \mathrm{~mA}$. В отличие от схемы, предложенной в работе [23], в нашем источнике в качестве компаратора использована микросхема K140UD8, которая проста в настройке и почти не требует навесных элементов. Преимуществами данного источника являются:

а) малый вес и габариты при достаточной выходной мощности;

б) автоматическая система зарядки конденсаторной батареи до заданного напряжения и его поддержания с точностью $0.5 \%$;

в) высокая надежность и КПД;

г) способность выдерживать короткое замыкание на выходе. 
Измерение зарядного напряжения производилось с помощью электростатического вольтметра С53 и высокоомного делителя напряжения с точностью $0.5 \%$.

При включении внешним пусковым импульсом управляемого разрядника - игнитрона IRT-3 - конденсатор разряжался через $\mathrm{EW}$ из меди $\varnothing 90 \mu \mathrm{m}$. В настоящей работе исследовался ЕВ в атмосферном канале, образованном $\mathrm{EW} \varnothing 90 \mu \mathrm{m}$, длиной $133 \mathrm{~cm}$ при начальной запасенной энергии $\sim 2.8 \mathrm{~kJ}$ и удельной напряженности электрического поля в канале $E_{\mathrm{c}} \approx 59 \mathrm{~V} / \mathrm{cm}$. Как показано в работе [6], для EW большой длины при пробое происходит монотонное расширение разрядного канала. Поэтому при оценке геометрии разряда правильнее выбрать среднюю скорость его расширения, а не значение его радиуса в какой-то определенный момент времени. Скорость расширения от удельной вложенной на единицу длины разряда энергии $\mathrm{W}$ зависит линейно [6]. В настоящей работе данный параметр определялся методом перехватывающего заземленного кольцевого электрода с внутренним диаметром, изменяемым в диапазоне $19-40 \mathrm{~mm}$. Фотография пробоя в канале при наличии перехватывающего кольца диаметром $20 \mathrm{~mm}$ приведены
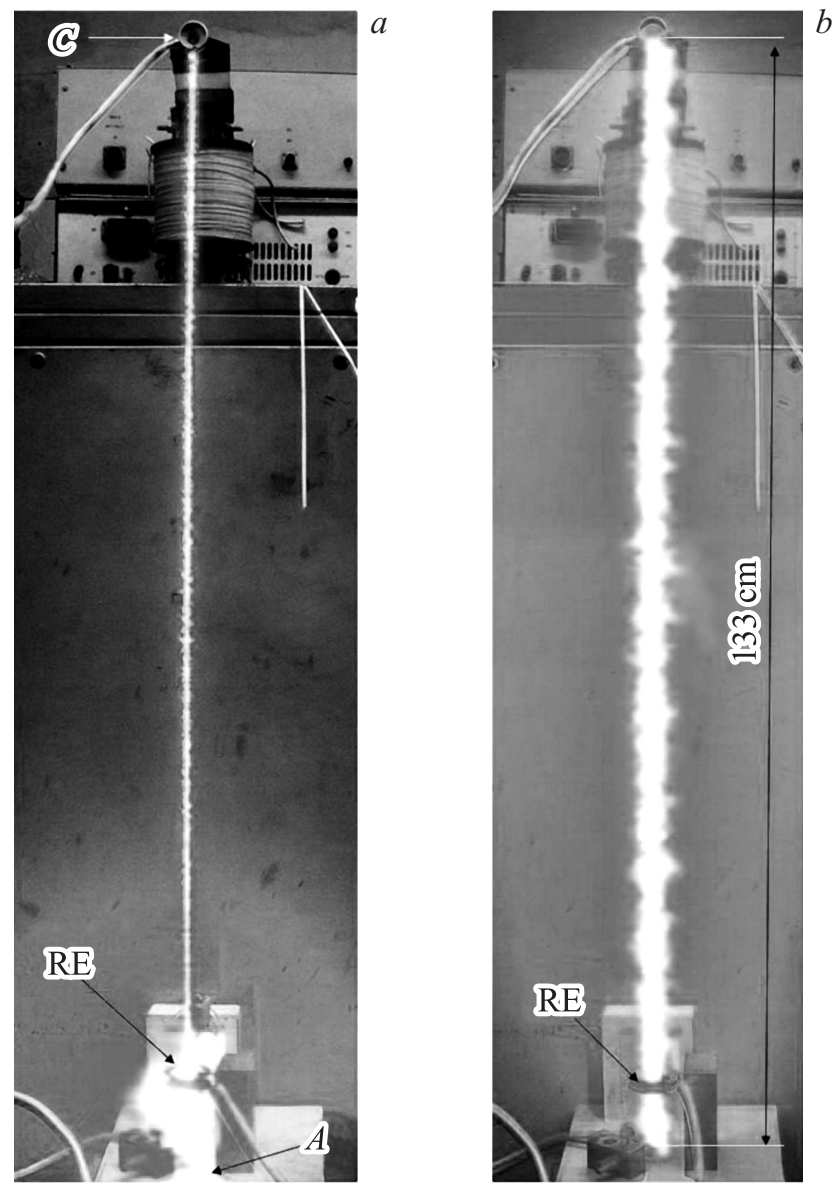

Рис. 2. Фотография электрического пробоя при вертикальном положении $\mathrm{EW}$ длиной $133 \mathrm{~cm}$ с перехватывающим кольцом заземления диаметром $20 \mathrm{~mm}(a)$, без перехватывающего кольца $(b) . A-$ анод, $C$ - катод.

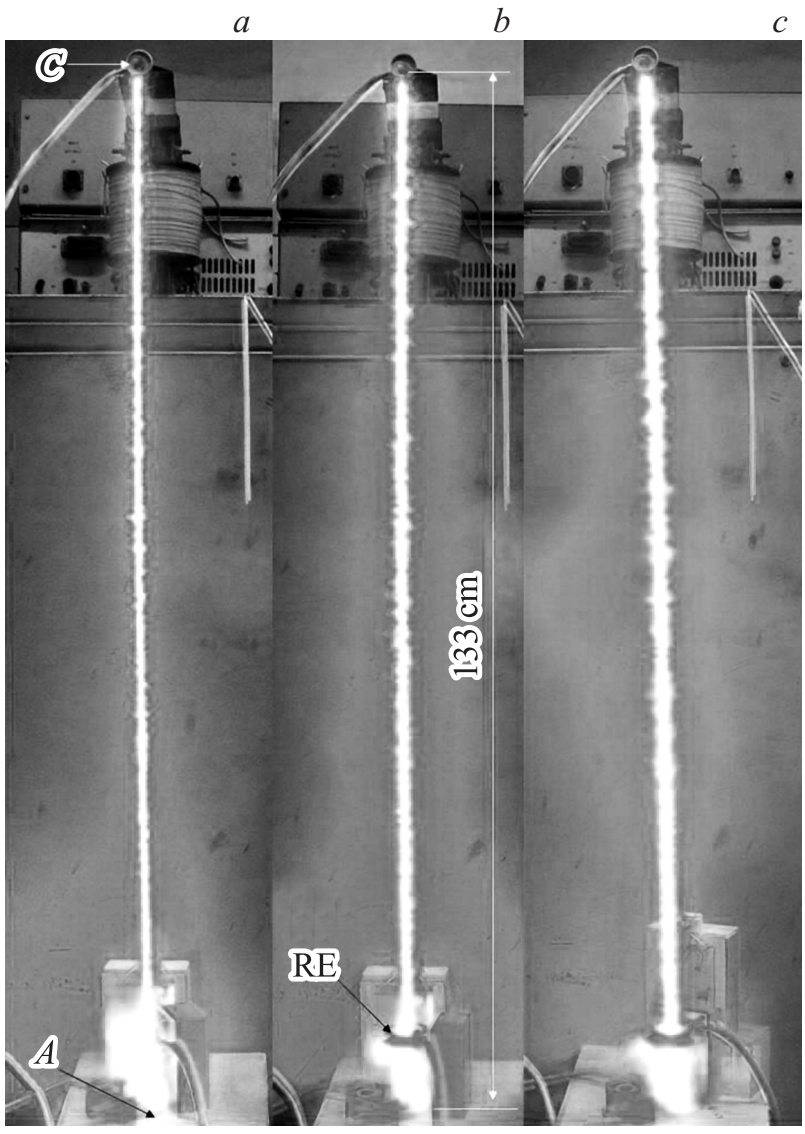

Рис. 3. Фотография электрического пробоя при вертикальном положении $\mathrm{EW}$ длиной $133 \mathrm{~cm}$ с перехватывающим кольцом заземления диаметром $24.5 \mathrm{~mm}(a), 28.3 \mathrm{~mm}(b), 32 \mathrm{~mm}(c)$. $A-$ анод, $C-$ катод.

на рис. $2, a$, а при его отсутствии на рис. 2, $b$. Фотосъемка проводилась через синий фильтр FS-1 совместно с нейтральным фильтром NS-9 с помощью цифровой камеры OLYMPUS C-5050ZOOM в ручном режиме. Параметры фотосъемки приведены в работах $[1,2]$. На рис. 2, $a$ видно, что при наличии перехватывающего кольца с внутренним диаметром $20 \mathrm{~mm}$ нагрев канала джоулевой энергией разрядного тока осуществлялся только до момента соприкосновения его с кольцом, соединенным с землей. Далее весь ток, минуя разрядник (игнитрон), замыкался на землю. Этот момент (интервал времени) $\Delta t$ фиксировался на осциллограмме тока поясом Роговского, который измеряет ток, протекающий по цепи через разрядник. Зная радиус кольца и интервал времени $\Delta t$, можно определить среднюю скорость расширения канала проводимости для данного перехватывающего кольца. При большом наборе колец с разными диаметрами можно получить (с определенной точностью) зависимость мгновенной скорости расширения канала. В нашем эксперименте мы ограничились набором из семи колец с внутренними диаметрами в интервале $19-40 \mathrm{~mm}$. 

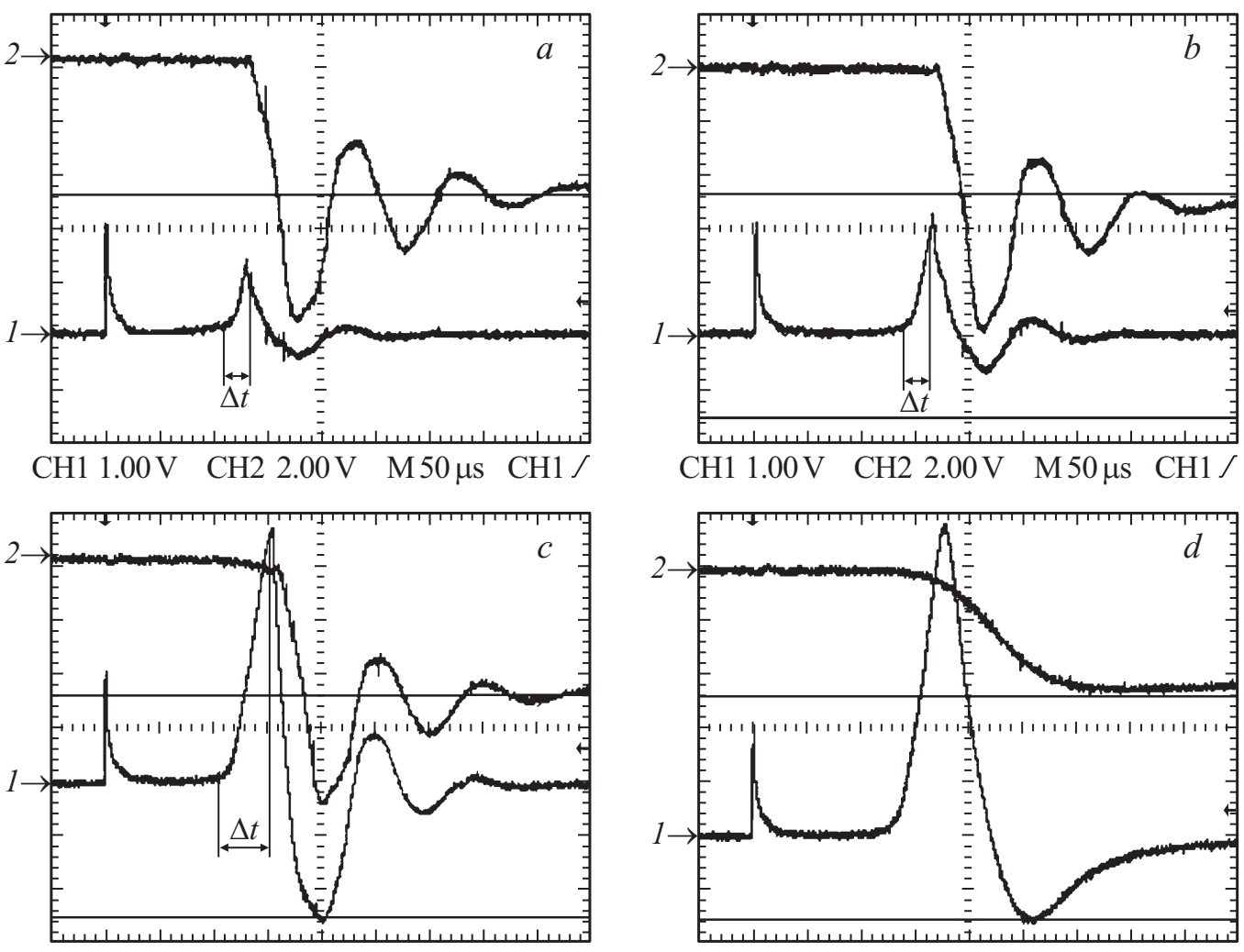

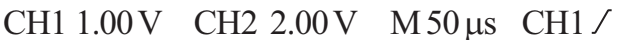
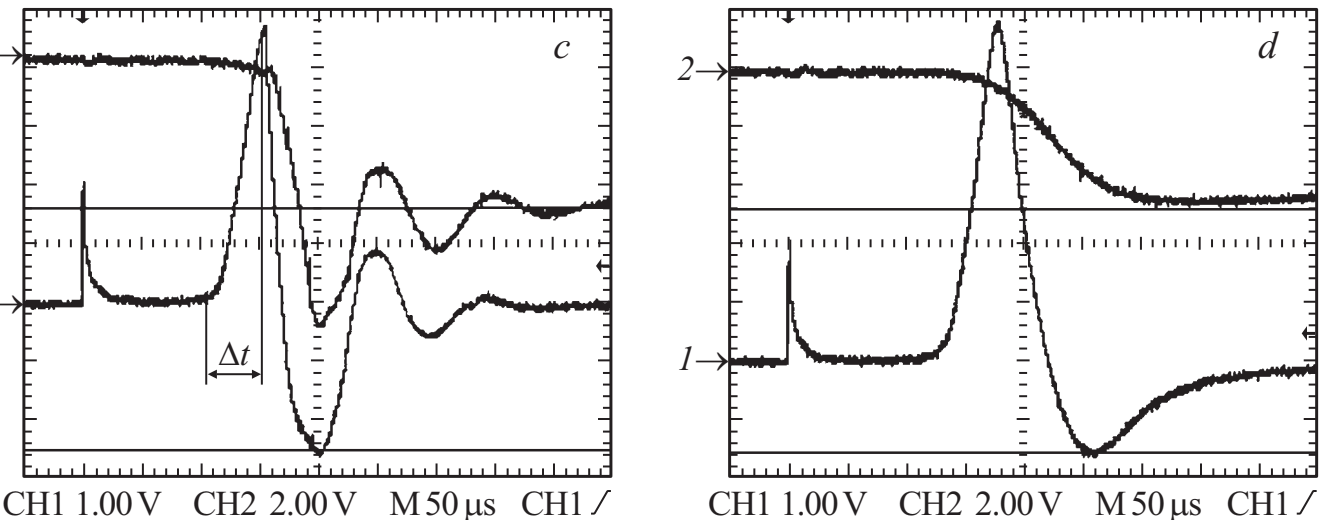

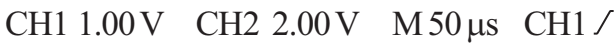

Рис. 4. Осциллограммы напряжения (2) и тока (1) управляемого пробоя при вертикальном положении EW длиной $133 \mathrm{~cm} c$ перехватывающим кольцом заземления диаметром $20 \mathrm{~mm}(a), 24.5 \mathrm{~mm}(b), 28.3 \mathrm{~mm}(c)$, без перехватывающего кольца $(d)$.

На рис. 3 приведены фотографии пробоя в канале при наличии колец диаметром $24.5 \mathrm{~mm}(a), 28.3 \mathrm{~mm}(b)$, $32 \mathrm{~mm}(c)$. С ростом диаметра кольца максимальный (конечный) размер проводящего канала увеличивался, что говорит о том, что после замыкания проводящего канала на заземленное кольцо поступление энергии в канал практически прекращалось.

На рис. 4, $a$ представлены осциллограммы напряжения (2) и тока (1) при наличии EB в канале EW $\varnothing 90 \mu \mathrm{m}$, длиной $133 \mathrm{~cm}$ при наличии кольца заземления диаметром $20 \mathrm{~mm}$. На рис. 4, $b, c$ представлены осциллограммы напряжения (2) и тока (1) при наличии EB в канале EW при наличии колец заземления диаметром 24.5 и $28.3 \mathrm{~mm}$ соответственно. На рис. $4, d$ представлены осциллограммы напряжения (2) и тока (1) при наличии EB в канале EW без перехватывающего кольца. С ростом диаметра кольца интервал времени $\Delta t$ до момента соприкосновения проводящего канала с кольцом, соединенным с землей увеличивался. Это хорошо видно на осциллограммах тока, значение которого резко уменьшалось в момент соприкосновения. Зная интервал времени $\Delta t$ и диаметр кольца, можно определить среднюю скорость расширения канала за данный интервал $\Delta t$.

Например, в эксперименте на рис. 3, с при удельной вложенной энергии $800 \mathrm{~J} / \mathrm{m}$ средняя скорость расшире- ния канала составила $\sim 400 \mathrm{~m} / \mathrm{s}$. Диаметр проводящей зоны канала $\sim 32 \mathrm{~mm}$.

В работе [6] показано, что в случае протяженных разрядов длиной $\sim 100 \mathrm{~cm}$ существует промежуток времени в течение первого квазипериода разрядного тока, когда разряд излучает как абсолютно черное тело и в течение которого происходит основной вклад энергии в разряд. В этом промежутке вкладывается $64 \%$ всей запасенной в батарее энергии. При удельном пробивном напряжение $E_{\mathrm{c}} \approx 59 \mathrm{~V} / \mathrm{cm}$ и максимальном диаметре канала пробоя нами сделана оценка максимальной вложенной удельной энергии в канал при пробое для запасенной в батарее энергии $\sim 2.8 \mathrm{~kJ}$, которая составила $\sim 1 \mathrm{~kJ} / \mathrm{m}$.

На рис. 5, $a, b$ представлены экспериментальные зависимости обратной величины скорости расширения канала $1 / U$ от $t^{1 / 2}(a)$, от радиуса канала $\mathrm{R}(b)$. На рис. 5, $c$ представлена экспериментальная зависимость радиуса канала $R$ от $t^{1 / 2}$. В отличие от результатов, полученных в [17] для чисел Mach's менее трех, данные зависимости получились линейными. Это совпадает с моделью сильной цилиндрической ударной волны, описанной в [18], при малых поперечных размерах источника ударной волны - взрываемой проволоки $\varnothing 90 \mu \mathrm{m}$. Линейная аппроксимация полученных экспериментальных кривых (показанная на рис. 5 штрихом) в область более высоких скоростей расширения приходит 

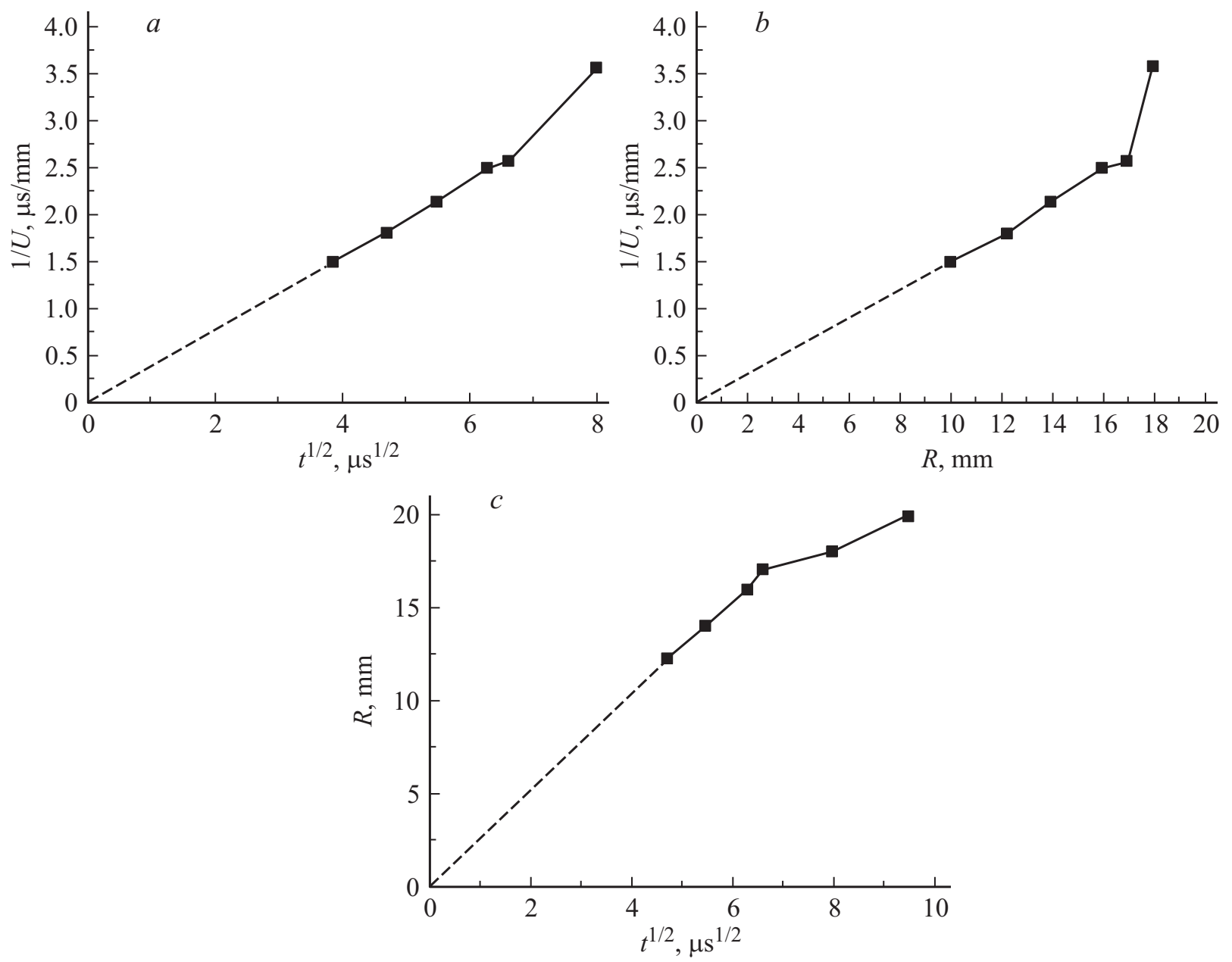

Рис. 5. Экспериментальные зависимости обратной величины скорости расширения канала $1 / U$ от $t^{1 / 2}(a)$, от радиуса канала $R(b)$, зависимость радиуса канала $R$ от $t^{1 / 2}(c)$.

в начало координат. Это говорит о том, что взрыв тонкой проволоки моделирует ,идеальную“ цилиндрическую ударную волну, что невозможно осуществить при взрыве цилиндрического заряда PETN (внешний радиус $15 \mathrm{~mm}$, внутренний радиус $4 \mathrm{~mm}$, высота $30 \mathrm{~mm}$ ), используемого в работе [17]. Из этого можно сделать вывод, что скорость расширения канала проводимости совпадает со скоростью распространения цилиндрической ударной волны в канале, образованной взрывом проволоки.

\section{Заключение}

В настоящей работе предложена новая экспериментальная методика определения скорости расширения канала проводимости в зависимости от времени распространения в экспериментах по управляемому пробою протяженных 0.6-1.9 m промежутков, образованных взрывом проволоки при максимальной вложенной удельной энергии в канал, превышающей $\sim 1 \mathrm{~kJ} / \mathrm{m}$. Используемая нами методика позволяет с точностью до 5\% процентов определить основные характеристики пробоя, а именно удельную вложенную энергию в канал разряда, динамику изменения удельного сопротивления разрядного промежутка. В модели равновесной плазмы эти данные позволяют определить максимальную температуру в канале разряда, что дает возможность более точного прогнозирования значений пробойного напряжения и удельного энерговклада при масштабировании управляемого пробоя на больших длинах. Полученные экспериментально зависимости скорости расширения канала $U$ и радиуса канала $R$ от времени распространения показали, что данные характеристики совпадают с точностью до $10 \%$ со скоростью и радиусом сильной цилиндрической ударной волны, которая образуется при взрыве проволоки при максимальной вложенной удельной энергии в канал, превышающей $\sim 1 \mathrm{~kJ} / \mathrm{m}$. Следует отметить, что в эксперименте была получена цилиндрическая „идеальная“ ударная волна, теория которой приводится в работе [18].

Авторы благодарны В.Р. Сороченко за обсуждение экспериментальных результатов, С.Ю. Казанцеву, Н.Б. Пономареву за помощь в работе. 


\section{Список литературы}

[1] Аполлонов В.В., Плетнев Н.В. // Квантовая электроника. 2012. T. 42. № 2. C. 130-139.

[2] Аполлонов В.В., Плетнев Н.В. // ЖТФ. 2013. Т. 83. Вып. 12. C. 67-80.

[3] Sinton R., van Herel R., Enrigh W., Bodger P. // J. Appl. Phys. 2010. Vol. 108. P. 053304.1-053304.4.

[4] Sinton R., van Herel R., Enrigh W., Bodger P. // J. Appl. Phys. 2011. Vol. 110. P. 093303.1-093303.4.

[5] Александров А.Ф., Зосимов В.В., Курдюмов С.П., Попов Ю.П., Рухадзе А.А., Тимофеев И.Б. // ЖЭТФ. 1971. Т. 61. Вып. 5(11). С. 1841-1854.

[6] Александров А.Ф., Рухадзе А.А. Физика сильноточных электроразрядных источников света. М.: Атомиздат, 1976. Изд. 2-е. М.: Книжный дом ЛИБРОКОМ. 2012. 184 с.

[7] Exploding wires. Vol. 1. NY: Plenum Press, 1959. (Взрывающиеся проволочки. Т. 1. М.: ИЛ, 1963. 342 с.)

[8] Exploding wires. Vol. 2. NY: Plenum Press, 1962. (Электрический взрыв проводников. Т. 2. М.: Мир, 1965. 341 с.)

[9] Базелян Э.М., Райзер Ю.П. Физика молнии и молниезащита. М.: Физматлит, 2001. 320 с.

[10] Абрамсон И.С., Гегечкори Н.М., Драбкина С.И., Мандельштам С.Л. // ЖЭТФ. 1947. Т. 17. Вып. 10. С. 862-867.

[11] Гегечкори Н.М. // ЖЭТФ. 1951. Т. 21. Вып. 4. С. 493-506.

[12] Ванюков М.П., Исаенко В.И., Хазов Л.Д. // ЖТФ. 1955. Т. 25. Вып. 7. С. $1248-1256$.

[13] Бобров Ю.К. // ЖТФ. 1974. Т. 44. Вып. 11. С. 2340-2347.

[14] Очерки физики и химии низкотемпературной плазмы / Под ред. Л.С. Полак М.: Наука, 1971. 436 с.

[15] Райзер Ю.П. // УФН. 1972. Т. 108. Вып. 3. С. 429-463.

[16] Зельдович Я.Б., Райзер Ю.П. Физика ударных волн и высокотемпературных гидродинамических явлений. М.: Наука, 1966.

[17] Birk M., Manheimer Y., Nahmani G. // J. Apll. Phys. 1954. Vol. 25. P. 1208.

[18] Lin S.C. // J. Appl. Phys. 1954. Vol. 25. P. 54-57.

[19] Bennett F.D. // Phys. Fluids. 1958. Vol. 1. P. 347-352.

[20] Bennett F.D. // Phys. Fluids. 1958. Vol. 1. P. 515-522.

[21] Герасименко Н.И., Грашина Н.А., Медведков А.Г., Мещеряков А.Б., Плетнев Н.В. // ПТЭ. 1988. № 1. С. 212-215.

[22] Гончаренко Г.М. Труды МЭИ. Вып. 45. Электроэнергетика. М.: МЭИ, 1963. 169 с.

[23] Насонов А.Г., Ланиман Л.С. // ПТЭ. 1984. № 2. С. 134-135. 